\title{
Relieving the Congestion around a School via Automatic Vehicle Identification Technology-Based Traffic Measures
}

\author{
Bo Li $\mathbb{D}^{1},{ }^{1}$ Zhi Yu $\mathbb{D},{ }^{2}$ Weiwei Sun, ${ }^{3}$ Kaiying Chen, ${ }^{3}$ and Teng Zhang ${ }^{3}$ \\ ${ }^{1}$ School of Automation, Guangdong University of Technology, \\ The Guangdong-Hong Kong-Macao Joint Laboratory for Smart Discrete Manufacturing, Guangzhou 510006, China \\ ${ }^{2}$ School of Intelligent Systems Engineering, Guangdong Provincial Key Laboratory of Intelligent Transportation System, \\ Sun Yat-Sen University, Guangzhou 510275, China \\ ${ }^{3}$ Fundway Technology, National Digital Application Demonstration Industrial Base, Guangzhou 510006, China
}

Correspondence should be addressed to Zhi Yu; stsyuz@mail.sysu.edu.cn

Received 10 February 2021; Revised 16 September 2021; Accepted 7 October 2021; Published 10 November 2021

Academic Editor: Yajie Zou

Copyright (c) 2021 Bo Li et al. This is an open access article distributed under the Creative Commons Attribution License, which permits unrestricted use, distribution, and reproduction in any medium, provided the original work is properly cited.

Recently, many parents drive their children to and from schools, leading to serious road congestion around the school gate. The school-related congestion is a special type of congestion caused by periodic impulsive aggregation of specific travellers for certain events. In this study, the individual long short-term traffic behaviours were reconstructed based on automatic vehicle identification (AVI) technologies. The cause and countermeasure of congestion around the service centers were identified through the individual behavioural properties. The vehicles that were primarily responsible for periodic impulsive aggregation congestion (PIAC) around the school gate were precisely targeted via a proposed vehicle grading clustering framework. The road management objectives were updated in the AVI data environment and it was found that only $3 \%-5 \%$ of the total number of vehicles passing by the school gate require specific management such as traffic enforcement activities. A series of traffic measures were formulated based on the results of vehicle grading clustering and achieved positive effects in a periodic impulsive aggregation area. It is an effective way to solve the PIAC by formulating management with different activity levels and resolutions for specific travellers. The methodologies and experience presented in this study may provide a useful tool for relieving such special type of congestion around other service centers faced with similar scenarios.

\section{Introduction}

The travel preferences of citizens have changed dramatically to the increasing reliance on private cars in the past few decades [1]. The accompanying local traffic congestion has become a hot spot of both public concerns $[2,3]$ and academic researches $[4,5]$. According to the frequency of occurrence, there are two types of congestion: (1) periodic congestion with fixed location and time interval and (2) nonperiodic congestion caused by accidental events [6]. Recently, a special kind of congestion called periodic impulsive aggregation congestion (PIAC), which including the characteristics of both periodic and nonperiodic, is observed. It is triggered by the periodic impulsive aggregation of specific travellers for certain events. The PIAC usually occurs in the area around the service centers such as schools, hospitals, and stadiums [7-9]. Compared with the periodic or nonperiodic congestion, the impact of PIAC is equally worrisome but has not been paid enough attention to detect and govern it. Hence, this highlights a need to identify and relive the PIAC around the service centers.

The current researches on traffic congestion relieving are mainly focused on the aspects of regional areas at a macrolevel, including road widening and reconstruction $[10,11]$, public transport priority [12, 13], signal optimization $[14,15]$, and traffic demand management $[16,17]$. Because the cause of PIAC differs from periodic or nonperiodic congestion, the application of these congestion management methods seems to be inefficient around particular service centers. The main reason is that the impact factors of PIAC 
need to be more systematically studied. The challenges include both identifying and relieving the PIAC around service centers under the condition of microstrategies failure. In addressing these challenges, personalized management based on individual traffic behaviours is urgently needed. Many studies deal with the identification of congestion in specific functional areas via data-driven approaches [18-20] but fail to present an integrated and effective solution to it. In practice, the rigid traffic demand of specific travellers renders the identification and management of PIAC around the service centers more difficulty. Three types of methods are commonly used to alleviate the congestion around the service centers: traffic planning adjustment method, new facilities construction method, and traffic guidance method. A typical representative of the traffic planning adjustment method is the spread of freestanding Emergency Departments around hospitals [21]. The results turn to be unsatisfactory and they are unable to alleviate the congestion around hospitals [22]. Hence, the traffic planning adjustment method may lose its effect around service centers. Although the new facilities construction method has the advantage of excellent adaptability to multiscenes, the costs are inevitably high. To improve the traffic conditions around the school, a multilayer underground car park for school staff and parents is specially built in Ningbo, China, which leads to much cost and limited effect [23]. Other effective and widely used types of relieving congestion method around the service centers are the traffic guidance method, which includes proactive vehicles route guidance [24], congestion pricing [25], and traffic demand assessment [26]. These methods mostly cover the regional macrolevel but lack intensive microcosmic analysis, especially the relation between the individual traffic behaviour of specific travellers and the evolution of the PIAC around the service centers.

The school-related congestion is a typical example of the PIAC that periodically occurs when school is open/close. To deal with school-related congestion, many scholars have made useful researches [27-29]. Lu et al. [27] concluded that driving-to-school trips are the main reason for school-related congestion by analyzing the temporal variations of citylevel traffic congestion and air pollution. Ermagun and Levinson [28] solved the school-related congestion by relocating the corresponding zones of schools into suburban areas. Rhoulac [29] developed a school transportation model based on questionnaires for the identification of potential customers of school bus service. However, in the past, the congestion mitigation strategies were generally limited by the accuracy and efficiency of the detectors. As a result, it was extremely difficult to formulate traffic measures for specific travellers according to their traffic behaviours with different activity levels and resolutions. With the ubiquitous application of automatic vehicle identification (AVI) technology, location-specific license plates can be collected with high real-time and accuracy from vehicles within the urban road network [30]. An et al. [31] used the location-specific license plates recognised by AVI not only to collect high real-time information from vehicles but also to estimate a lane-based traffic arrival pattern. Given its rich spatial-temporal information, AVI data has been widely used to provide more advanced intelligent transportation services [32-34]. But a gap is observed between the wide deployment of AVI detectors and the potential applications in the identification, internal cause analysis, and alleviation of the congestion around the service centers. The AVI technology provides a good foundation for efficient and precise vehicle detection at fixed locations and further an opportunity to understand the PIAC around service centers at a microlevel. In recent years, there is an increasing tendency for families to move outside of city centers to avoid congestion, unsafe neighbourhoods, or high housing prices. Hence, many service centers are located at a significant distance from peoples' homes and travel to these locations can lead to excessive periodic impulsive aggregation congestion.

In this study, we focus on the use of AVI data to deal with the PIAC around school by accurately identifying the vehicles that are primarily responsible for the congestion formation near the school gate. Specifically, we use a convolutional neural network $(\mathrm{CNN})$ to capture the long shortterm features of individual traffic behaviour images, and then the extracted features are used as the input to an agglomerative hierarchical clustering algorithm. According to the observation of individual road travel time and clustering results, we further evaluated the contribution of different types of vehicles to the PIAC formation around the case school. Finally, based on the name list of vehicles that were primarily responsible for PIAC, a series of mitigation strategies were designed and implemented, which prove the effectiveness of the proposed methodologies. The comparison experiment in Xuancheng City 6th primary school, China, shows that the proposed methods provide effective and sustainable ways to personalized management for the PIAC at a microscopic individual level. One of the main advantages of this study is that it is purely data-driven, accurately targets specific travellers with different activity levels and resolutions, and hence is particularly appealing to the PIAC alleviation in the area with restriction to the new equipment cost, management budget, and land resources. This study also found that the evaluation of road traffic conditions should be based on the clustering results of individual traffic behaviour. The vehicles that are affected by those mainly responsible for leading to congestion are the real representative of road traffic state, rather than total vehicles observed on the road.

\section{Preview of the Automatic Vehicle Identification}

2.1. AVI System. With the development of machine vision and data transmission technologies, the emphasis on traffic detection has shifted from centralised metering to the precise detection of individual travellers. The centralised measurement of flow, speed, and density is usually a sampling observation of all participants on the road. This means that the centralised measurement can only represent the overall traffic situation; however, it cannot be detailed to the actual traffic behaviour of individuals. For obtaining different traffic behaviours on the same road, the missing part of centralised metering is the detection of vehicles' 
identification, while some types of individual detection such as global positioning system (GPS), Bluetooth, and mobile signalling contain the identity label of the vehicle, and the low sampling and penetration rate severely limits their potential for individual traffic behaviour analysis.

The AVI system provides a good balance between the identity detection of vehicles and their sampling rate. In recent years, the AVI system has been widely deployed at signalised intersections in urban areas of China. The entire AVI system consists of three components: front detection, data transmission, and data processing units. As shown in Figure 1, the front detection unit contains a slow-motion video camera, a high-definition (HD) camera, and auxiliary lighting devices. The slow-motion video camera is used to determine whether a vehicle is moving in the detection area and the HD camera for capturing photos of vehicles entering the detection area. The cameras and corresponding secondary light sources are usually set on an L-shaped gantry, $6 \mathrm{~m}$ above the ground. The horizontal distance between the stop line (the center point of the captured image) and the gantry is $20-25$ meters. The camera detects the moving vehicles and the colour of the signal light. The visual field of the camera contains both the intersection entrance and signal lamp. The captured pictures are used as evidence of a vehicle running a red light. Hence, the front detection unit of an AVI system captures almost all vehicles passing through a specific lane including license plate number, vehicle type, detection time stamps, and the location of the camera.

2.2. AVI Data. The data transmission unit is composed of a communication device such as a switch and an optical transceiver. All front equipment collections are uploaded to the data processing unit via the $100 \mathrm{M}$ Ethernet and fibre optic network. The data processing unit is responsible for the identification, access, query, storage, and management of the detection videos and pictures of vehicles. When a vehicle is detected, the tracking program is started and the position of the vehicle is continuously traced in the subsequent frame. Until the vehicle leaves the detection area, the position of the vehicle, the colour of its signal light, and the HD camera can be identified to capture a picture for identification. This is different from the detection of vehicle's arrival by other types of sensors. The detection area of the AVI system has a finite capacity of the queuing vehicles, usually $2-3$ vehicles. The acquisition of AVI data corresponds to two situations: (1) only identify the vehicles in front of the queue (from 1st to 3 rd) during the red interval; (2) the rest of vehicles in the queue will be detected as the queue discharging after the traffic light turns green; that is, when green light turns on, we can get the information about whether there is a queue and whether a new vehicle is entering the detection area and if so, the timestamp of it. The vehicle identification process involves three steps:

(i) S1: vehicle enters the detection area, initiating the camera

(ii) S2: a snapshot is captured (capture rate $\geq 99 \%$ ) and the license plate is located (location rate $\geq 98 \%$ ) (iii) S3: skew correction, character segmentation, and recognition are performed on the captured license plate image (recognition rate $\geq 95 \%$ )

Next, the plate number and timestamp of the vehicle passing through the detection area are obtained using the AVI system. These information and device ID are uploaded to the central database in real time by the data transmission unit. The structures of the AVI data and the explanation of each field are provided in Table 1.

Table 1 indicates that the front detection units are marked at the location level, which is a precoding SSID for a signalised intersection or an interesting point. The minimum unit of AVI detection is at the level of the lane for the specific point that the camera is deployed. According to the performance of the vehicle identification processes, the AVI system achieves a good balance between the sampling and recognition rates. Hence, the AVI data around the case school is used as the original dataset for our study.

2.3. Virtual Fusion AVI Detection. We define "AVI perfect detection" as a layout of AVI cameras with the following characteristics: (1) for a given road section or sections, there are AVI cameras deployed at the upstream and downstream intersections, (2) all license plates of vehicles can be identified without missing vehicles or incorrect detection, and (3) there is no branch road or parking place between any pair of adjacent AVI cameras. Although it is difficult to fulfil this perfect AVI detection scheme in a real traffic scenario, multiple groups of AVI cameras can realise "relative perfect detection" with a missing rate of less than $1 \%$ in some parts of the road sections.

An AVI system helps acquire information on every vehicle passing through the detection area wherein AVI cameras are deployed. The information includes license plate number, vehicle type, detection time stamps, and the location of the camera. The AVI data are essentially a departure record for the vehicle, that is, the time stamp for the vehicle that enters the next road section. The characteristics of the AVI system provide us with an opportunity to achieve the "relative perfect detection."

Although it is not possible to identify the license plates of all vehicles with a camera in a real traffic scenario, our field survey suggests that the recognition rate provided by the AVI system under normal working conditions can exhibit an accuracy greater than $90 \%$. Hence, when a vehicle passes continuously through the detection areas in adjacent locations, the probability of recognition failure is less than $1 \%$. Based on this property, two AVI cameras are deployed at the midpoint of the road section to form a detection group with the cameras located at the upstream intersection. As shown in Figure 2, the AVI cameras at the upstream intersection and the camera in the road constitute a fused virtual group for AVI detection. It can be regarded as the "relative perfect" recognition of the identities of all passing vehicles in the cost of travel time measurement. Owing to a backpropagation feature of the queue at the downstream intersection, the virtual fusion AVI detection does not significantly affect the recognition of the traffic state for the rest of the road section. 


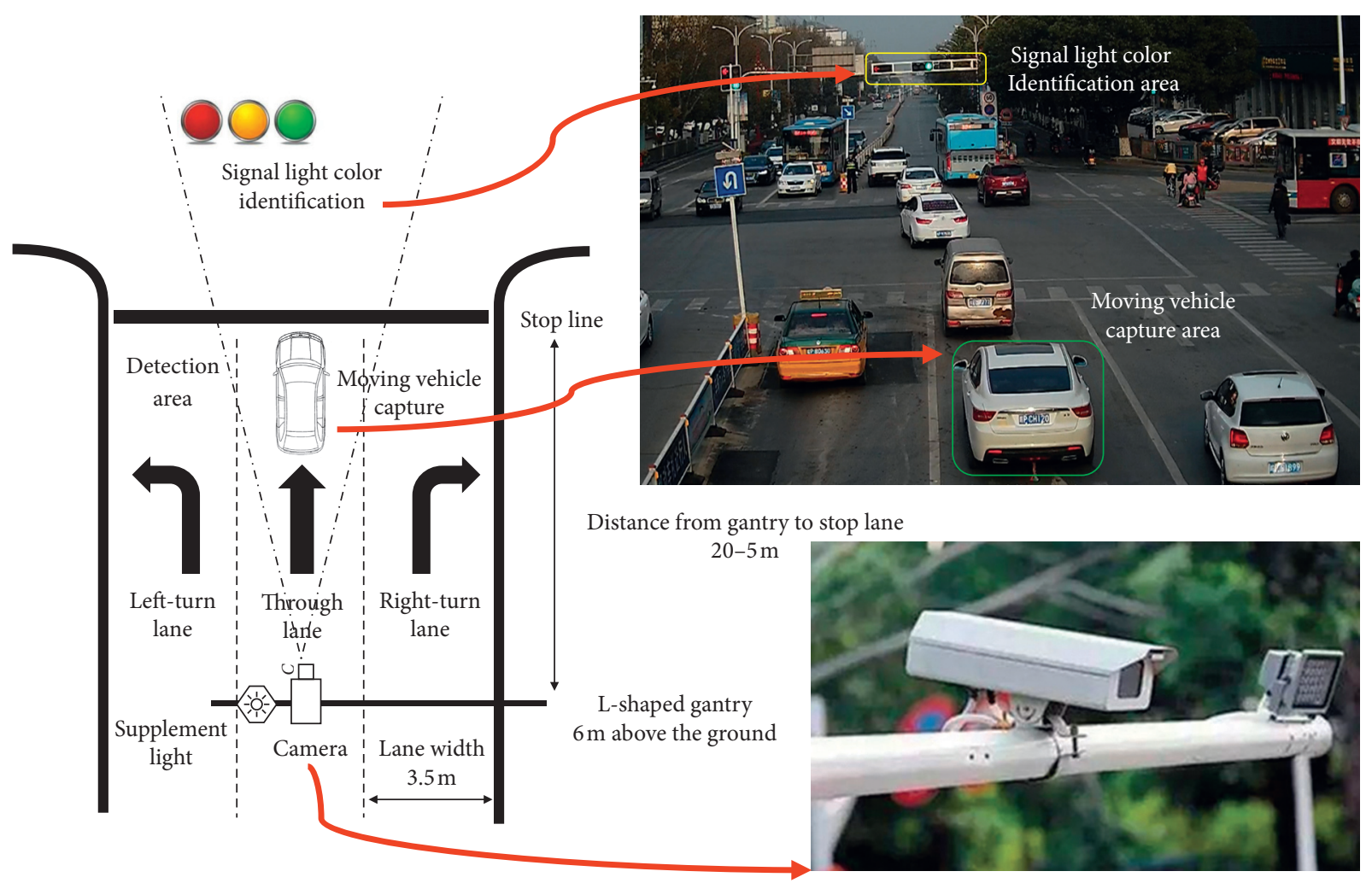

Figure 1: Front detection unit of an AVI system.

The "relative perfect detection" provides a solid foundation for studying the clustering of key vehicles and for evaluating the performance of new policies and management strategies.

\section{Materials and Methods}

3.1. Study Area and Data Preparation. The case school adopted in this paper is located on Aofeng Road, the main road in the central urban area of Xuancheng, $230 \mathrm{~m}$ away from the intersection of Aofeng and Jincheng Road on the west side, and $458 \mathrm{~m}$ away from the intersection of Aofeng and Wanxi road on the east side. The road section in front of the school gate is a bidirectional road with four motor vehicle lanes and two cycle lanes. Eastward is the direction out of the city. The AVI cameras are deployed at three locations on the road, including the upstream and downstream signal intersections and the sidewalks in front of the school gate. These AVI cameras provide abundant data resources for our research.

There are residential communities and functional departments on the road section around the school. Hence, large-scale construction, widening, or reconstruction of traffic facilities cannot be performed in the study area. The school has more than 2500 students and 110 teaching and administrative staff. The school ending time ranges between $16: 55$ and $17: 15$. Figure 3 indicates the surroundings around the case school. The road can be divided into four links (links 1-4 as shown in Figure 3) based on the deployment locations of the AVI cameras.
In our study, the training set contains 3283584 AVI data from 8 May to 31 October 2019, and the testing set consists of the AVI data and survey data (ground truth) around the case school during 21-31 October. The AVI data are provided by 121091 vehicles that appeared on the road in front of the case school between 15:30 and 18:00 every day. The dataset covers 117918 private cars, 1434 taxis, and 1739 vehicles of other types (bus, trucks, police car, etc.) with a total of 869923 road travel records.

3.2. Overview of the Method. Figure 4 presents an overview of the research framework for the periodic impulsive aggregation congestion. The study applied an AVI data-driven approach to developing the methods to reconstruct individual traffic behaviours and identify and relieve the PIAC. Based on the analysis, mitigation strategies with different activity levels and resolutions for specific travellers were formulated. Finally, the proposed methods were evaluated by the field test around the case school.

3.3. Road Management Objectives in AVI Data Environment. Under the condition of "relative perfect detection," the license plates and time stamps of vehicles passing the detection area are detected with a high penetration rate. In this paper, the dynamic capacity is defined as the capacity at the bottleneck of the road section, that is, the location in front of the case school gate. The static capacity refers to the carrying capacity of the road, and it is roughly estimated from the 
TABLE 1: AVI data fields description.

\begin{tabular}{lcc}
\hline Filed name & Field type & Field description \\
\hline SSID & VARCHAR2 & Device ID for a specific location \\
HPHM & VARCHAR2 & License plate \\
HPZL & VARCHAR2 & Type of license plate \\
(02 for private car) \\
JGSJ & DATE & Detecting timestamps \\
CDBH & NUMBER & Camera ID for a specific lane \\
FX & VARCHAR2 & Direction of the vehicle \\
\hline
\end{tabular}

space between passenger car units and the lane length of the road.

Suppose that $\Omega_{S}$ denotes the topological space of the road network. An arbitrary vehicle $i$ and its position $x$ in the road network at time $t$ are recorded as $x_{i}(t)$. Let the set $X_{i}(T)$ be the location set of vehicle $i$ during the time interval $T$; that is, $x_{i}(t) \in X_{i}(T)$. Thus, for an arbitrary road $S \subset \Omega_{S}$, $\chi_{S}\left(x_{i}(t)\right)$ is the characteristic function of road $S$, and it can be defined as

$$
\chi_{S}\left(x_{i}(t)\right)= \begin{cases}1, & x_{i}(t) \in S, \\ 0, & x_{i}(t) \notin S,\end{cases}
$$

where $\delta_{x_{i}(t)}(S)=\chi_{S}\left(x_{i}(t)\right)$ can be regarded as the Dirac delta function of vehicle $i$ remaining in an arbitrary position $x$ on $\operatorname{road} S$ at time $t$. Then, for time $t$, the number of vehicles on the road $N_{S}(t)$ is the Dirac measure of road $S$ at time $t$. Based on equation (1), we thus have

$$
N_{S}(t)=\sum_{i} \int_{s \in S} \delta_{x_{i}(t)}(S) \mathrm{d} s .
$$

Hence, the AVI detection of vehicle $i$ constitutes a specific observation of the Dirac measure at upstream or downstream intersections. Let $t_{i}^{u}, t_{i}^{d}$ be the time stamps for vehicle $i$ entering and leaving the road, respectively. Then, for an arbitrary time $t \in\left[t_{i}^{u}, t_{i}^{d}\right]$, the Dirac measure of an arbitrary position of vehicle $i$ on the road will be 1 . Therefore, the average travel time on road $S$ during a time interval $T$ is derived as

$$
\phi(S, T)=\frac{\sum_{t_{i}^{u}} \varphi_{i}^{S}}{\sum_{i} \int_{t \in T} \delta\left(t-t_{i}^{u}\right) \mathrm{d} t},
$$

where $\varphi_{i}^{S}=t_{i}^{d}-t_{i}^{u}$ is the total travel time of vehicle $i$ from entering to leaving road $S$. It includes both the travel time on the road and the delay when queuing at a downstream intersection.

Similarly, the traffic demand of road $S$ during time interval $T$ can be defined as

$$
Q_{S}(T)=\sum_{i} \int_{t \in T} \delta\left(t-t_{i}^{u}\right) \mathrm{d} t .
$$

Let $L_{S}, C_{S}$ be the static and dynamic capacities of road $S$. Then, the objective of road management in the AVI data environment can be defined as shown in the following equation:

$$
\begin{aligned}
& \min \phi(S, T) \\
& \text { s.t. } N_{S}(t) \leq L_{S}, t \in T, S \subset \Omega_{S}, \\
& \quad Q_{S}(T) \leq C_{S}, S \subset \Omega_{S} .
\end{aligned}
$$

The objective function indicates that the object of road management is to minimise the average travel time in the AVI data environment.

The classical methods for individual traffic behaviour analysis are mostly driven by low-dimensional and discrete traffic observations, which cannot fully capture the characteristics of individual traffic behaviour in different dates and periods. The AVI data provide opportunities to establish the long short-term individual traffic behaviour profile with multidimensional information. In this study, the historical AVI data and corresponding trip information compose the single trip vectors to construct the individual traffic behaviour image for specific travellers. A single trip vector mainly includes static attributes such as vehicle type, date type (workdays or holidays), and dynamic information such as road section, lane, and travel time, as shown in Figure 5. Due to the fact that the length of AVI data sequences may not be consistent, the main purpose of establishing multidimensional individual traffic behaviour image (Figure 5) is to standardize the fixed-length expression of the observation AVI data with variable length, to be used as the model input of individual long short-term traffic behaviour feature vector extraction.

Compared with the one-dimensional AVI data sequence, the multidimensional individual traffic behaviour images reflect the distribution characteristics of individual traveller in different dimensions (dates and periods). Data standardization is carried out in the form of high-dimensional images, which makes the original low-dimensional and linear traffic behaviour correlation characteristics transform into high-dimensional and regional traffic characteristics with local time-domain (including dates and periods). Furthermore, we can depict more discriminative individual traffic behaviour profiles from a higher dimension. As shown in Figure 5, this study established a multidimensional individual traffic behaviour image with a license plate number as the label. The individual travel time is expressed and reorganized in a matrix by date and period, and the single trip vector can be a high-dimensional element in the matrix.

In practice, individual vehicles may not generate AVI data in a particular date or period. In this study, a zerovector point is used to represent the situation that individual vehicles do not travel in multidimensional traffic behaviour image. Specifically, an individual travel set $S_{b}$ containing $q$ single trip vectors can be expressed as shown in the following equation:

$$
\begin{aligned}
& S_{b}=\left\{m_{1}, m_{2}, \ldots, m_{i}, \ldots, m_{q}\right\}, \\
& m_{i}=\left\{z_{i 1}, z_{i 2}, \ldots\right\},
\end{aligned}
$$

where $m_{i}$ is the $i^{\text {th }}$ trip vector for a specific vehicle and $z_{i}$ corresponds to the depth information in $m_{i}$. Based on the individual travel set $S_{b}$, this study reorganizes $S_{b}$ according to 


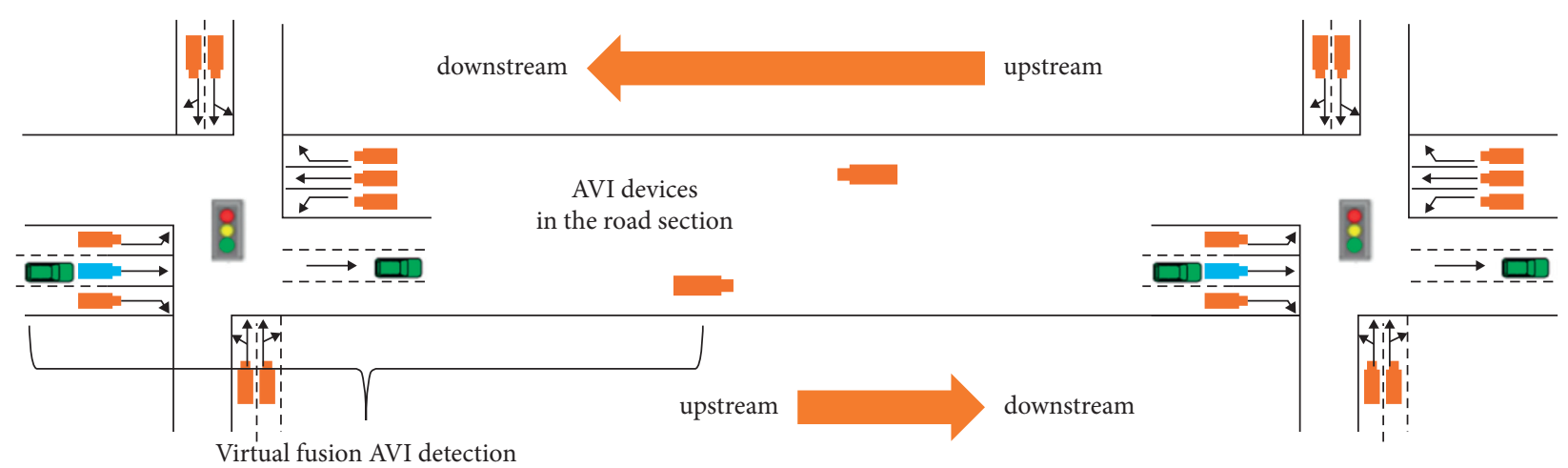

FIgURE 2: Virtual fusion AVI detection.

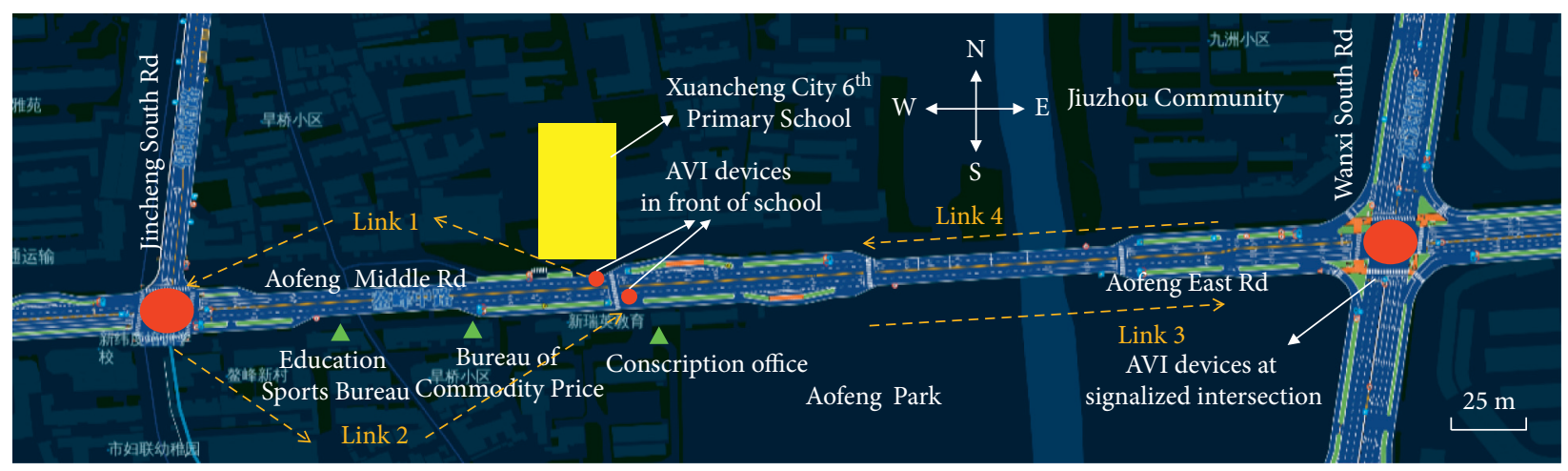
A Governments
- Device location
Case School

FIGURE 3: Surroundings of the case school.

Input

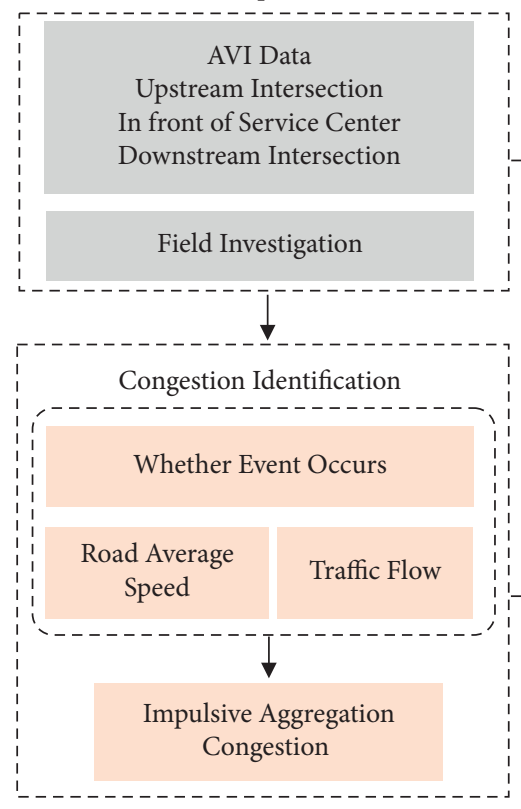

Analysis
Analysis

Congestion Responsibility Allocation

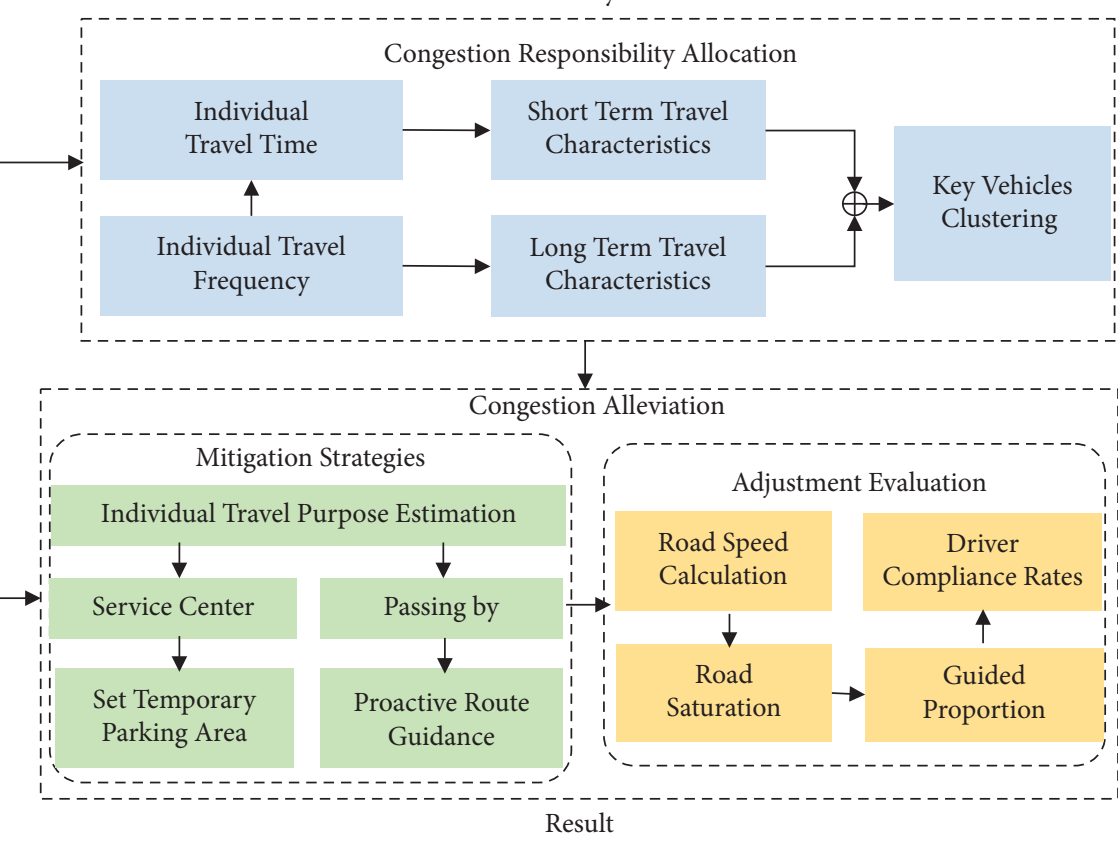

FIgURE 4: Flowchart for the methodology for mapping AVI data and relieving PIAC. 
the date and time information and converts it into a tensor matrix $S_{M}$. Each element in the matrix corresponds to a single trip vector in the original travel set $S_{b}$ and then normalizes all elements in the matrix $S_{M}$, as shown in the following equations:

$$
\begin{aligned}
S_{M} & =\left[\begin{array}{cccc}
m_{1} & m_{2} & \cdots & m_{u} \\
m_{u+1} & m_{u+2} & \cdots & m_{u+u} \\
\vdots & \vdots & \vdots & \vdots \\
m_{u(v-1)+1} & m_{u(v-1)+2} & \cdots & m_{u v}
\end{array}\right], \\
\widehat{S}_{M} & =\frac{\left[S_{M}-\min \left(S_{M}\right)\right]}{\max \left(S_{M}\right)-\min \left(S_{M}\right)},
\end{aligned}
$$

where $u, v$ denote the width and height of the traffic behaviour image, with $u v=q$, corresponding to travel date and time, respectively. The $\min (\cdot)$ and $\max (\cdot)$ represent the minimum and maximum travel time extraction function from individual travel set $S_{b}$. Thus, the multidimensional individual traffic behaviour image containing license plate label information is established.

We choose a multilayer convolutional neural network (CNN) as the tool to capture the long short-term traffic behaviour features. Considering that the convolution layer can retain the shape of input individual traffic behaviour image, the correlation of individual single trip vector in two dimensions of both date and timestamp can be effectively identified. Besides, through the sliding window mechanism, the convolution layer can perform a cross-correlation operation between the submatrix at different positions of the individual traffic behaviour image and the convolution kernel, which effectively reduces the dimension of the image and avoids overabundant parameters. The output of the convolution layer adopts a ReLU activation function and inputs a $2 \times 2$ maximum pooling layer to reduce the position sensitivity of the convolution layer. The convolution layer block is composed of the above basic units, and the long short-term traffic behaviour features extraction network (shown in Figure 6) used in this study is composed of two convolution layer blocks and one fully connected layer, to obtain the feature vectors containing the association of individual long short-term traffic behaviour. These feature vectors are used as input for vehicle grading clustering.

3.4. Vehicle Grading Clustering. While all vehicles passing by the school gate contribute to the development of schoolrelated congestion, most of them suffer from it. Vehicles that illegally occupy the motorway for long time cause PIAC around the school and benefit from their illegal behaviour simultaneously. This implies that the goal of picking up children is achieved through illegal traffic behaviours. The differences in travel purposes make traffic behaviours of the vehicles passing on the road section significantly different.

The AVI detection environment allows accurately identifying vehicles primarily responsible for the PIAC around case school. With the relative perfect detection environment provided by virtual fusion AVI detection, the vehicles passing by the school gate can be classified as highfrequency vehicles and occasional vehicles based on their travel frequency during the period after school. In this paper, the clustering time interval refers to the $30 \mathrm{~min}$ periods before and after school ends.

High-frequency vehicles are defined as those vehicles that appear more than five times on the same road in the statistical period within a week or more than three times during the clustering time interval of workdays. Simultaneously, the high-frequency vehicles can be divided into two clusters according to their travel purposes: (1) passing vehicles that must pass the road in front of the service centers every day after work are called Type A high-frequency vehicles affected by PIAC (Type A vehicles for short), and (2) aggregation vehicles that cluster around the service centers for a specific purpose are regarded as Type B high-frequency vehicles caused PIAC (Type B vehicles for short).

Figure 7 shows the framework of key vehicle clustering. For picking up children, Type B vehicles appear during the school days and disappear on weekends or during holidays. Conversely, because of the different travel purpose of Type A vehicles, their presence is not affected by winter and summer vacations. Therefore, for the key vehicles clustering around case school, it is necessary not only to count the travel frequency of different vehicles every week but also to distinguish the two types of high-frequency vehicles according to their travel frequencies during school days and nonschool holidays.

Figure 8 presents the clustering algorithm flowchart with the input long short-term travel traffic behaviour features extracted by the CNN network. In this study, the average link with log-likelihood distance is used as the similarity measurement for hierarchical agglomerative clustering. Algorithm 1 lists the pseudocode of the proposed framework in Figure 7. It starts by initializing each high-frequency vehicle in $\Omega$ that has appeared near the school into $N$ clusters. Secondly, the similarity matrix for the classes in $\Omega$ is calculated. Then, the two closest samples are merged as a new class on the basis of average-link principle and the similarity matrix is updated. Algorithm 1 repeats the above processes until two types of clusters left and returns the clustering license plates group $P_{1}^{T} \in C_{1}, P_{2}^{T} \in C_{2}$.

There are obvious differences in short-term traffic behaviours between these two types of high-frequency vehicles. In general, the parents' vehicles arrive near the school before class is over and do not leave until the children come out of school. Hence, even if congestion occurs, the travel time for parents' vehicles is significantly longer than that for commuter vehicles. Thus, two types of high-frequency vehicles can be distinguished accurately. The license plate of the vehicle and the travel time is extracted from the AVI data captured at the upstream and downstream intersections and the pavement in front of the school gate. The travel time of an individual vehicle includes both the samples passing through the entire road section and the samples in subsections. The abnormally large values of the travel time samples can be used as the basis for distinguishing parents' picking-up and commuter high-frequency vehicles. 


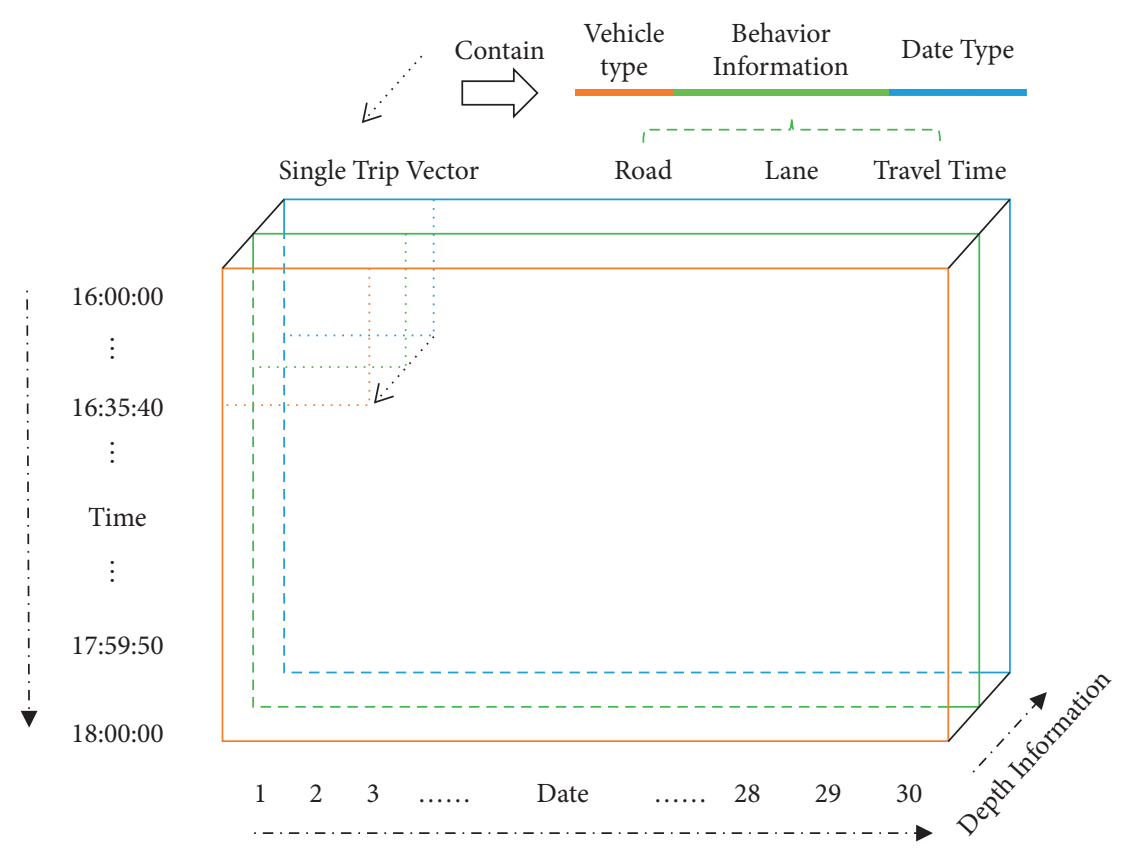

FIGURE 5: Multidimensional individual traffic behaviour image.

\section{Results and Discussion}

4.1. Identification of PIAC around the Case School. To identify the PIAC around the case school, a comparison of the traffic flow and average speed calculated from AVI data for school days and summer holidays is designed. The purpose of this comparison is to determine whether parents' pickup trips will generate additional traffic pressure and lead to PIAC around the school. For comparative experiments, we choose August 15 to represent a summer holiday, and the contrastive school day is May 16. The average speed and traffic flow are estimated at 5 min intervals from 16:40 to $17: 40$ every day, using the AVI data from three different deployment locations in front of the case school.

After school, a temporary intersection is formed on the pavement in front of the school gate. Traffic conflict is caused by the students' crossings and east-west traffic flow. Since there is no pedestrian signal in front of the school gate, a traffic police officer must be present at the center of the crosswalk every afternoon to manage the flow of students and vehicles and avoid spillback in links 1 and 2. The traffic in front of the school gate is disordered, and congestion occurs every day when the school ends; this makes it the most stubborn traffic problem on the main road in the central area. Remarkably, staff members' vehicles mixed with the flow of students, which makes traffic condition worse.

In our study, the traffic flow is derived from virtual fusion AVI detection on the road. According to the working characteristics of the AVI detector, the detection time stamps of vehicles are very close to the time at which they pass the stop line of the intersection. Thus, detection time can be regarded as the approximate starting time for most vehicles entering the next road section. However, for vehicles located in front of the queue at the signalised intersection, their detection time stamps are very different from the time stamps for passing the stop line. Therefore, the estimated average speed for the road section is inevitably affected by the delay for vehicles at the signalised intersection downstream of the road section.

To alleviate the effects of signal control, we consider the AVI cameras in front of the case school gate as the downstream point and those at the signalised intersection as the upstream point. The data are used to estimate the travel time of the individual vehicle in the corresponding section, and then, we calculate the average speed of the section as a representative of the actual travel speed. We use the average speed of link 4 as the average speed of the road section. There are three reasons for this selection: (1) based on historical data, there is more traffic volume on the case road travelling from east to west during the evening peaks; (2) the total length of the case road is $688 \mathrm{~m}$, and the length of link 4 is $440 \mathrm{~m}$, which better reflects the traffic condition than link 2; and (3) for most vehicles in link 4, the travel time will not be affected by downstream delays. Figure 9 presents a comparison of traffic flow and the average speed (see equation (3)) on the case road on weekdays.

The results show that the traffic flow (solid lines) between $16: 40$ and $17: 40$ is the same irrespective of whether it is a school day or a summer vacation. The traffic volume for every $5 \mathrm{~min}$ interval is generally between 50 and 60 vehicles, and the hourly volume is 600 to 700 vehicles. A significant difference is observed in the average speed (dotted lines) between the school days and summer holidays. During the school days, the average speed of the road is about $18 \mathrm{~km} / \mathrm{h}$, and the average road speed in 5-minute intervals can be as low as $9 \mathrm{~km} / \mathrm{h}$. The minimum speed appears at $17: 15$, about $20 \mathrm{~min}$ after school. In contrast, the average speed is $24 \mathrm{~km} / \mathrm{h}$ during summer vacation, which is about $33 \%$ higher than 


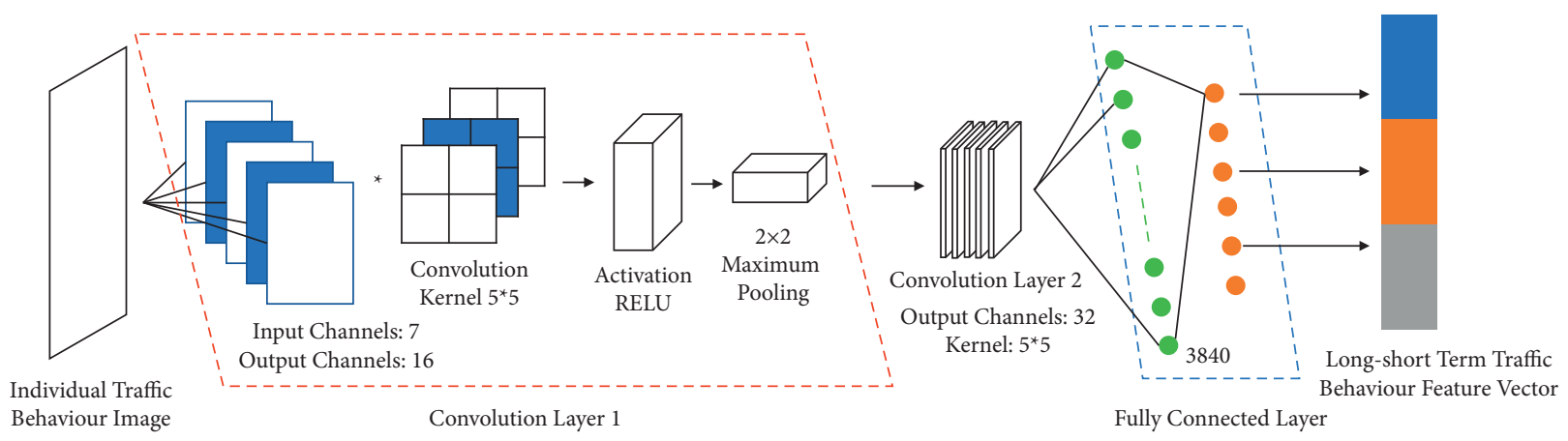

FIgURE 6: Long short-term travel traffic behaviour feature vector extraction network based on CNN.

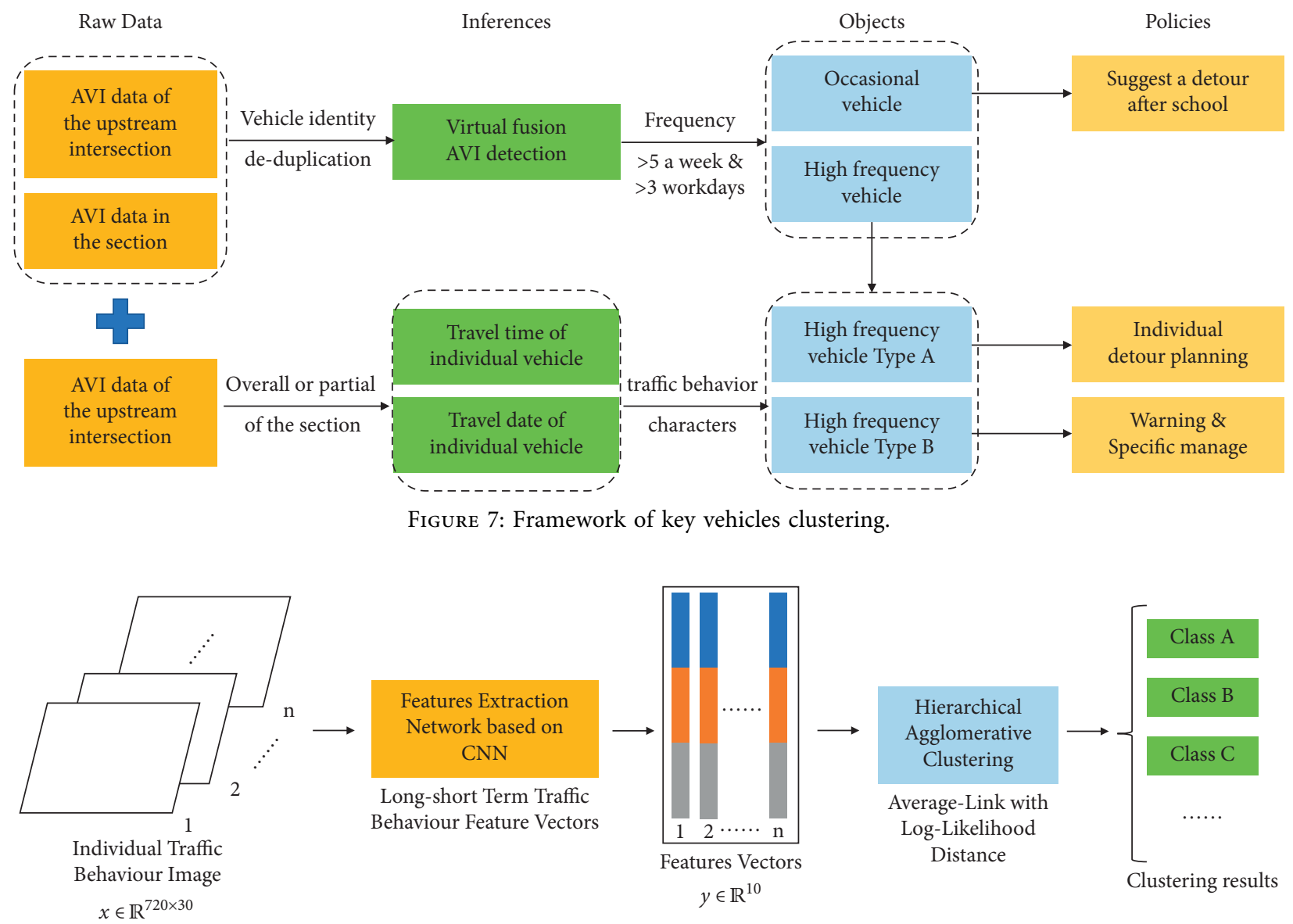

FIGURE 8: The clustering algorithm based on long short-term travel traffic behaviour features.

that on school days; the minimum speed observed in $5 \mathrm{~min}$ periods is $22 \mathrm{~km} / \mathrm{h}$. These findings are very interesting. The comparison of traffic flow and average road speed indicates that although the traffic demand is similar, the traffic situation differs widely between school days and summer holidays. Parents' pickup trips may generate little traffic pressure; however, the congestion around the school still occurs. The travel time is a direct observation of traffic behaviour. To explore the causes of congestion during school days, the individual travel time distributions are calculated through AVI data from 16:30 to $17: 30$ every day on school week and summer holiday week. The summer holiday week starts on August 12 and ends on August 18, and the school days are from May 13 to May 19.

Figure 10 presents the histograms of travel time distributions on school days and summer holidays; the distributions of travel time samples are different. The maximum travel time on the road observed during August 12-18 is $387 \mathrm{~s}$ and most travel time samples are distributed in the range of $30-150 \mathrm{~s}$. In contrast, 506 abnormally large travel time samples appeared during May 13-19, which were greater than the maximum travel time observed during the summer holidays. Besides, the travel time samples during May 13-19 were distributed in the range of 50-250 s. 
Input: a set of feature vectors $\Omega$ for individual behaviour portrait, the number of elements $N$, with log-likelihood distance measure.

Output: license plate numbers of two types of vehicles $P_{1}^{T}, P_{2}^{T}$.

(1) $P_{1}^{T}, P_{2}^{T} \leftarrow \varnothing$

(2) $C_{1}, C_{2}, \ldots \leftarrow$ Classify each element in $\Omega$ into a class $C_{i}$

(3) Calculate the similarity matrix for the classes in $\Omega$

(4) for $k=1$ to $N$ do

(5) $\quad C_{i} \leftarrow$ Merge the two closest samples as a new class;

(6) $\quad C_{j} \leftarrow$ The other existing classes in $\Omega-C_{i}$;

(7) $M_{i} \leftarrow \sum_{x \in C_{i}, y \in C_{j}} D_{\log }(x, y) / n_{i} * n_{j}$ Update the similarity matrix;

(8) Until two types of group $C_{1}, C_{2}$ left.

(9) end for

(10) return the clustering license plates group $P_{1}^{T} \in C_{1}, P_{2}^{T} \in C_{2}$

Algorithm 1: Agglomerative hierarchical clustering.

Particularly, these abnormally large travel time samples often appear after school and disappear at the same time interval during summer vacation. A reasonable explanation is that some vehicles stopped in the road section during the 30 min periods before and after school ends. Considering that there is no parking lot around the case school, curb parking becomes parents' only choice to pick up their children.

Abnormally large travel time samples from AVI data indicate that parents parking at curbs is not an occasional behaviour of few parents. The different distributions of travel time on school days and summer holidays show that curb parking demands are related to the event of school ending. Hence, school-related congestion is directly related to the work and rest of the case school. In other words, the schoolrelated congestion is caused by periodic impulsive aggregation of parents for children picking-ups.

4.2. The Clustering Results of Vehicles Responsible for PIAC around the Case School. Some may say that since the main reason for school-related congestion is illegal curb parking, the solution is quite simple: remove the illegally parked vehicles that are not supposed to be there, and follow traffic rules. Indeed, curb parking on the motor lane during peak traffic is forbidden by traffic rules. But for parents that arrive at the school for children picking-ups, their parking demands are rigid and reasonable. Although PIAC around the school is caused by parents' curb parking, as a traffic manager, we should consider how to meet these reasonable traffic demands, rather than roughly enforce traffic rules. Further, curb parking behaviour can be attributed to the fact that parking demands near the school were not considered during traffic planning decades ago. The key point to relieve PIAC around the case school is accurately distinguishing the vehicles of the parents from all other vehicles that appear on the road during school days. As a result, personalized management with different activity levels and resolutions for travellers based on individual traffic behaviours are urgently needed.

Clarifying the parking demand of parents is the basis of adjusting to existing traffic planning schemes. Individual travel time can be used as the main parameter to distinguish different traffic behaviours. As shown in Figure 10, abnormally large travel time samples were observed during school days. It is necessary to determine the critical value of travel time, that is, the critical value calibration of abnormally large travel time, to classify different traffic behaviours such as queue for passing and curb parking. We calculated 26934 road travel time samples in front of the case school through 104997 AVI data on school days from May 8 to July 8, 2019. According to these measurement results, the probability cumulative curve of the road travel time on school days near the school is fitted as presented in Figure 11.

The coordinates of the travel time inflection point are $368(96.5 \%)$. It indicates that $96.5 \%$ of travel time samples are less than $368 \mathrm{~s}$ on the school days from May 8 to July 8 . This can be used as a critical value of abnormally large travel time to distinguish curb parking from other traffic behaviours. Combined with the long-term travel characteristics, the parents' vehicles that are primarily responsible for school-related congestion are targeted.

Table 2 summarizes the clustering results of high-frequency vehicles on the basis of 1 , that is, Type A and Type B vehicles. In particular, this study did not establish individual traffic behaviour image for vehicles that occasionally appear on the case road and classified them as Type $C$ vehicles. As shown in Table 2, the proportion of high-frequency vehicles in a month is only about $3 \%-5 \%$, which means that most vehicles on the case road are occasionally affected by the periodic event of school ending. The long short-term travel properties of Type A vehicles indicate that they are commuter vehicles and often affected by the PIAC around case school. In other words, Type A vehicles are not responsible for the PIAC formation. The school ending time is 16: $55-17: 15$. The long short-term travel preference of Type B vehicles proves a direct correlation to the picking-up behaviours by parents' vehicles. In this research, the ratio of different types of vehicles' travel time to the sum of individual travel time was used as an indicator to measure the contribution of PIAC formation. Although the proportion of Type $\mathrm{B}$ vehicles is very low, the congestion contribution reaches $57.4 \%$, suggesting that they are primarily responsible for the PIAC around the school gate. 


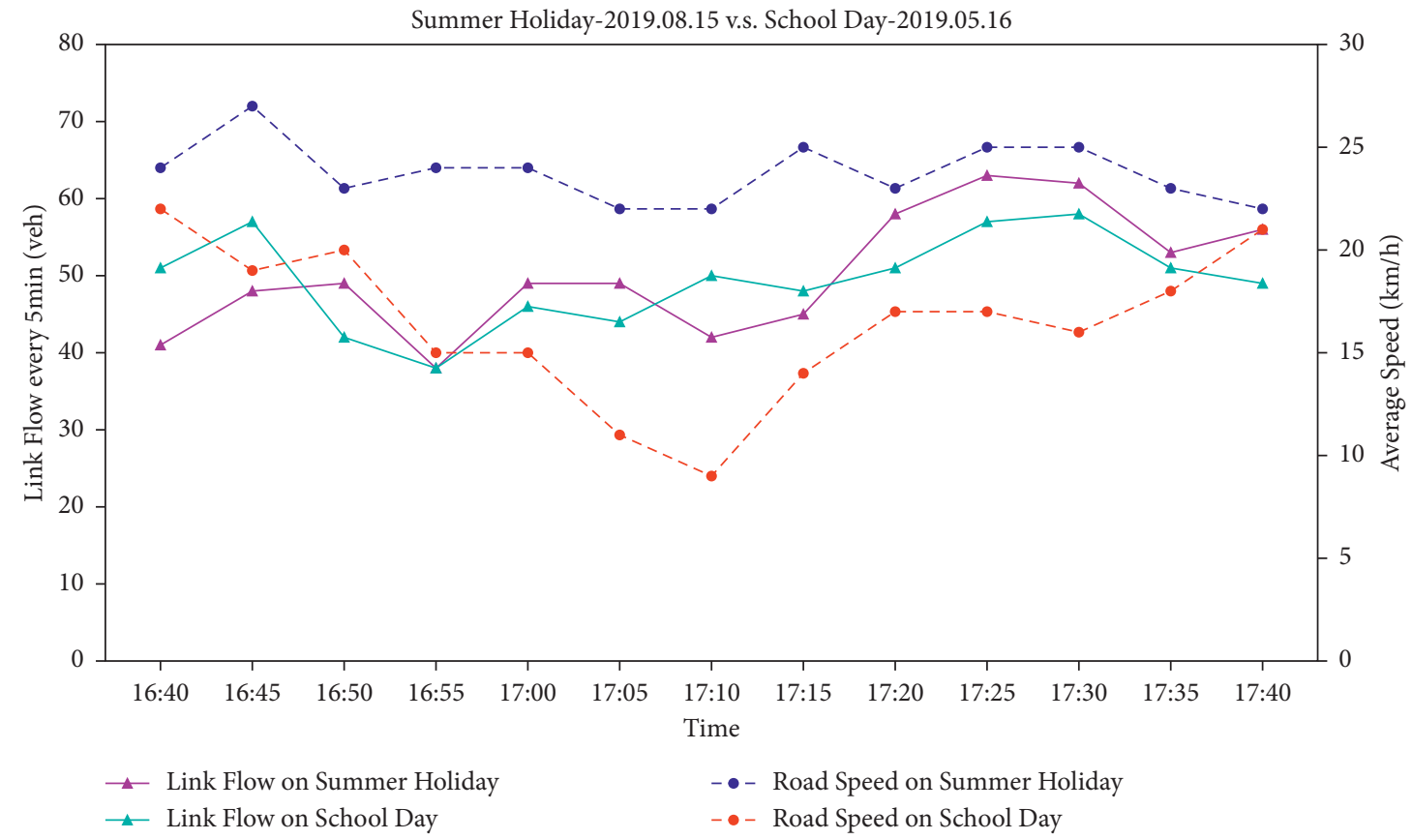

Figure 9: Traffic flow and average speed on weekdays.
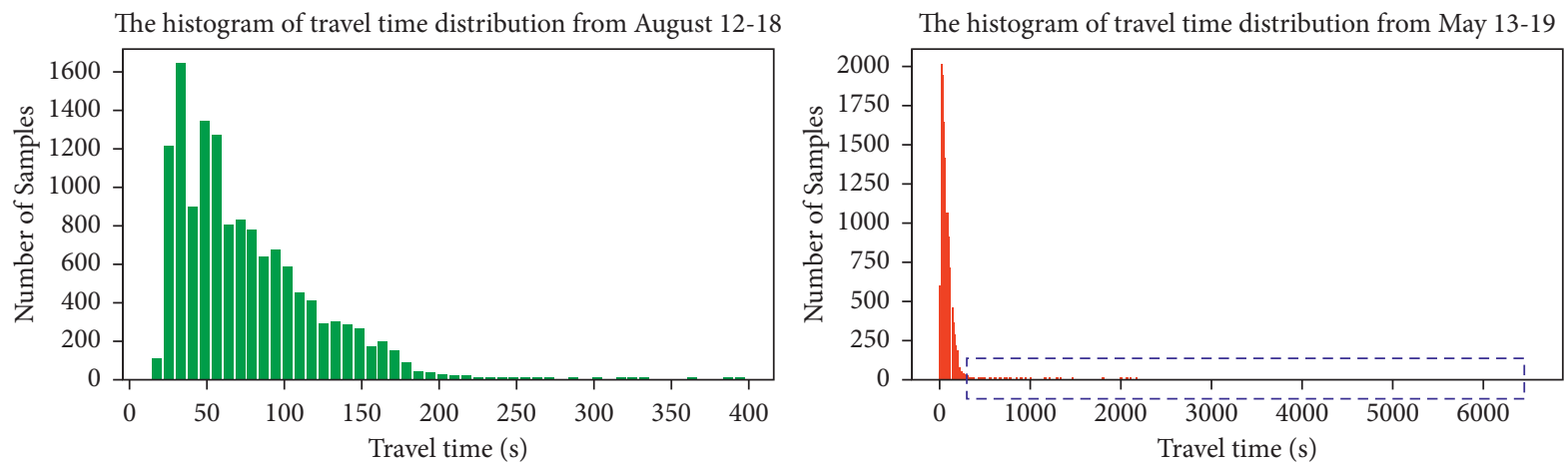

The local travel time (>400) distribution from May 13-19

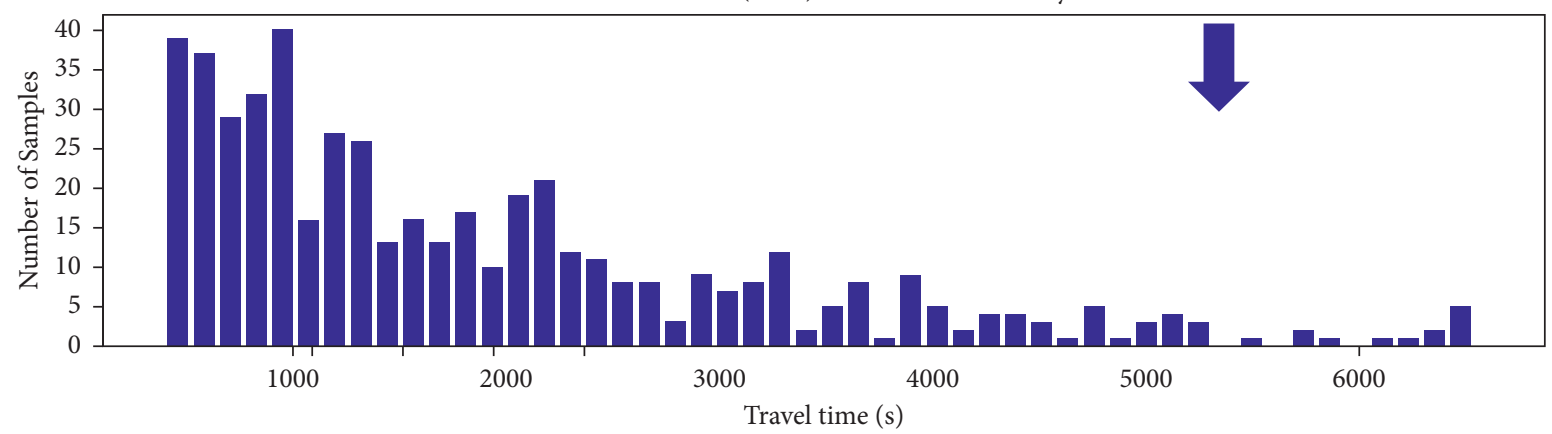

FIgURE 10: The comparison of travel time distributions on different weeks.

Table 3 lists the statistical results for parents' picking-up vehicles on the case road from September 1 to October 31, 2019. According to the travel frequency characteristics of these vehicles, we further found that about $15 \%-20 \%$ of the parents pick up their children every school day. These vehicles become the most active element in the PIAC formation around the case school and need to be managed especially. There are 19 school days in September with an average of 5 pickup trips for each vehicle. In October, the number of high-frequency vehicles decreased to 165 . There are 12 school days in October with an average of 7 pickup trips for each vehicle. The results indicate that the number of Type B vehicles decreases; however, the frequency of pickups increases in October. 


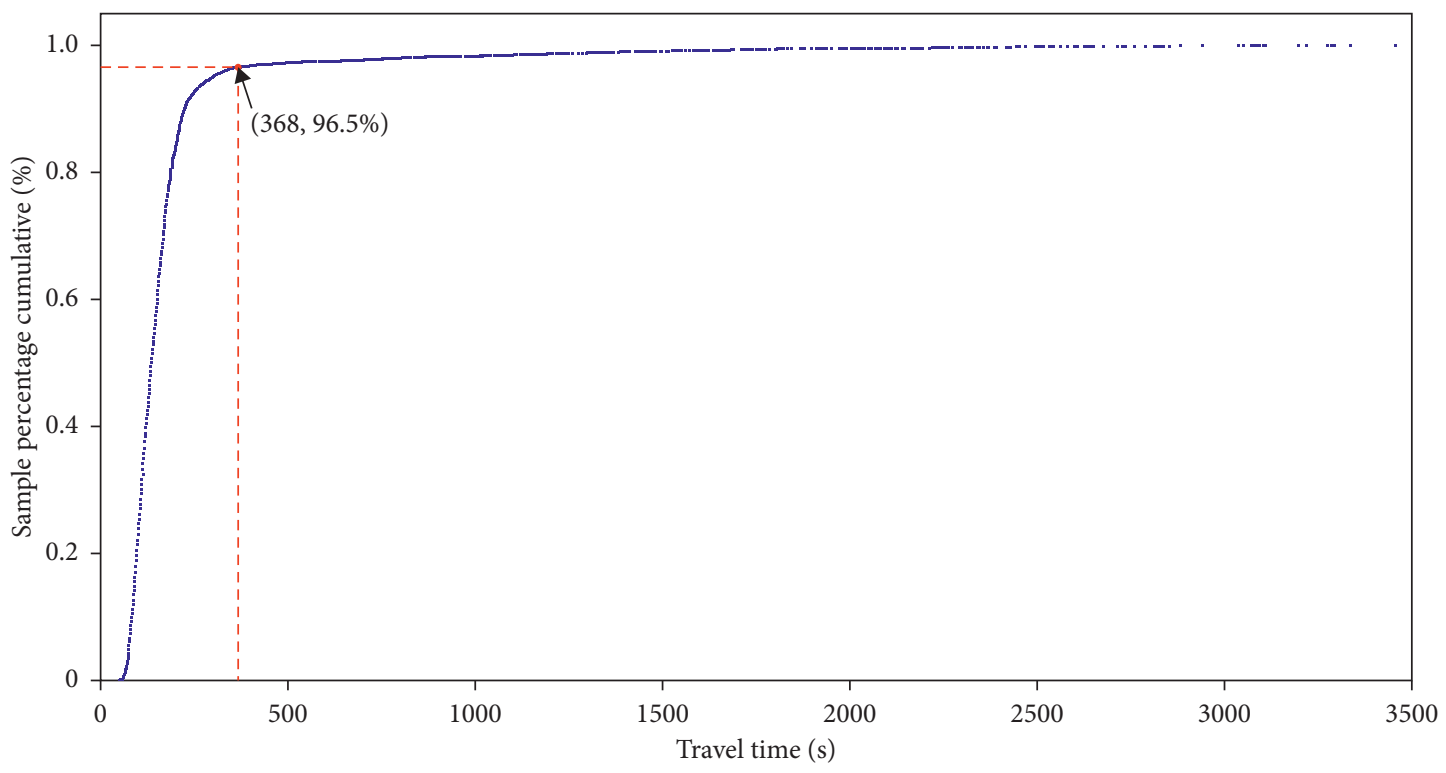

FIgURE 11: Probability cumulative curve of road travel time.

From the results of key vehicles clustering (in Table 2), we realised that the proportion of Type $\mathrm{B}$ vehicles present after school is very small. Hence, there is a good chance of improving the road capacity and relieving the PIAC around the case school by meeting the parking demands of very few parents under the condition of avoiding unnecessary new constructions as far as possible. Traffic engineers and researchers joined forces with the traffic police department, the administrative section of the 6th school, and the municipal officials. Through multisectoral collaboration, a series of adjustments have been adopted to relieve the congestion on the road in front of the case school gate during the evening peak periods.

\subsection{Mitigation Strategies for PIAC around the Case School.} With the analysis in the previous sections, we found that Type B vehicles are largely responsible for the congestion around the school during evening peak periods. Due to the limited availability of parking spaces near the case school, parents have no choice but to curb parking and this is the primary cause of PIAC around the case school. From the results of vehicle grading clustering, we also found that the proportion of Type $B$ vehicles during the school ending period is very small (about 6\%). Therefore, only the traffic behaviours of Type B vehicles must be changed, suggesting that there is a good chance of improving the road capacity and easing the PIAC around the case school. Next, according to the objective function in equation (5), the policy-making principles for developing traffic policies with more humanisation and lower management costs will be introduced.

When making adjustments involving the surrounding areas of service centers, the following principles should be considered:
(1) Define the goals of management: the management should aim to change the behaviour of both students and parents, that is, change from "Type B vehicle's long time waiting for picking up" to "students' short time waiting for being picked up." The specific goal is to reduce the impact of picking-up behaviour rather than forbidding pickups.

(2) Reduce the management cost: multiple measures can be applied to alleviate the PIAC around service centers, such as building more parking lots, opening multiple school gates, or organising parents as traffic control volunteers, but the process of implementation will entail considerable manpower and material resource investments and result in high management costs. Hence, it is necessary to minimise the management cost for congestion control.

(3) Clarify the management objectives: the AVI system provides support for the mitigation strategies with multiple levels and resolutions. While developing hierarchical management strategies for vehicles appearing on the same road in the same period, the implementation of adjustments could also bring about changes in public opinion. Special attention should be paid to the coordination between travellers and managers.

Based on the above principles, the detour suggestion SMS is sent to Type A vehicles and the vehicles passing by case school occasionally are not managed. To meet the parking demands of the parents, a temporary parking area is demarcated. It is very important to determine the capacity of the parking area. The capacity is determined by the number of Type B vehicles from our vehicle grading clustering. This indicates that the temporary parking area does not need to be very large. Instead, it only needs to be 
TABLE 2: Clustering results on the case road from September 1 to October 30.

\begin{tabular}{lcccccc}
\hline \multirow{2}{*}{ Clustering results } & \multicolumn{2}{l}{ Number of each type } & \multicolumn{3}{c}{ Long short-term travel properties } & \multicolumn{2}{c}{ Congestion } \\
& September & October & Date preference & Time preference & Travel time interval (s) & contribution (\%) \\
\hline Type A & 1049 & 763 & Workdays & Random distribution & $50-250$ & 5.8 \\
Type B & 262 & 165 & School days & $16: 30-16: 45$ & $600-3000$ & 57.4 \\
Type C & 33159 & 28273 & Random distribution & Random distribution & $50-250$ & 36.8 \\
\hline
\end{tabular}

Table 3: High-frequency vehicles on the case road from September 1 to October 30.

\begin{tabular}{lcc}
\hline Dates & Number of picking-up vehicles & Picking up every school day \\
\hline $2019.09 .01-09.30$ & 262 & 40 \\
$2019.10 .08-10.31$ & 165 & 34 \\
\hline
\end{tabular}

sufficient for the parents who pick up students frequently. Using the AVI data and proposed key vehicle clustering framework, the managers obtained a list of Type B vehicles and used it as a basis to clarify the scale of the temporary parking area.

As presented in Figure 12, a temporary parking area is defined using the bicycle lane opposite the case school gate. The inner reason for this choice is that the area is convenient for vehicles to disperse quickly. The designated area is in link 3 (in the out-of-town direction, shown in Figure 2), and it has a limited influence on nonmotorised vehicle traffic. As strategies include an open letter published by the school to advocate students to use public transit over private cars, detour advice to the drivers of Type A vehicles from traffic police and special actions to prevent vehicles from illegally occupying the motor lane for a long period.

4.4. Evaluation of the Adjustment Implementation. Adjustments and key vehicle management strategies were implemented on October 20, 2019. To evaluate the overall effect of the practical application, the average road speed and road saturation during the school end period (16:30-17:45 every day) for a week before and after the implementation of the fine-tunings were estimated. The traffic near the case school was significantly improved.

Figure 13 shows the scene near the pavement in front of the school gate at the same time before and after the implementation of the fine-tunings. The period chosen for comparison is 2019.10.17 versus 2019.10.24, which is a Thursday, 10 minutes before school ends. As shown in Figure 13(b) of field scene in front of the case school, on the right side of the road, many parents are sitting on electric bikes to wait for picking up their children. And the road is wet, which indicates that it has just rained. As in Figure 13(a) for comparison, there are also electric bikes parked in the motorway on the right side of the road. The comparison of Figure 12 demonstrates that, even under the condition of bad weather, our proposed AVI-based adjustments still have a good performance on the traffic conditions improvement.
Figure 14 presents the comparison of the hourly traffic volume before and after the adjustment on the basis of equation (4) in front of the case school during the workdays and weekends (16:30-17:30 every day). It indicates that traffic demand of the case road is similar during workdays and weekends, ranging from $700 \mathrm{pcu} / \mathrm{h}$ to $800 \mathrm{pcu} / \mathrm{h}$, far from the design traffic capacity (about $1000 \mathrm{pcu} / \mathrm{h}$ for a two-lane road). The traffic volume after adjustment is even larger (during 10.22, 10.23, and 10.25). That is to say, the traffic conditions in the surrounding area that affected the case school are relatively stable. Hence, the specific effect of the adjustment measures can be evaluated by comparing the average road speed of the road section with the consideration of the factors such as weather, holidays, and other traffic conditions in the surrounding area.

The average road speed was calculated through the AVI data in link 4. As presented in Figure 15, the overall average speed in front of the case school during the school ending period increased by $40 \%$ (from $14.2 \mathrm{~km} / \mathrm{h}$ to $19.7 \mathrm{~km} / \mathrm{h}$ ), and the observed minimum average speed rose by $106 \%$ (from $6.95 \mathrm{~km} / \mathrm{h}$ to $14.33 \mathrm{~km} / \mathrm{h}$ ). This indicates the average speed of vehicles passing by during the school ending period is significantly increased. Further, the traffic in the direction into the city achieved desirable outcomes after fine-tuning.

The main issue for the direction out of the city is the spillback caused by PIAC around the case school in link 1 . Road saturation can be used to describe the traffic in this subsection. The number of vehicles in link 1 at an arbitrary time point was estimated using equation (2). Combined with the length of lanes in link 1 , the road saturations were further obtained.

As shown in Figure 16, there is little difference in road saturation at weekends. Significant diversity was observed during workdays before and after fine-tuning from October 14 to 27,2019 . After satisfying the parents' parking demands, the saturation of link 1 ranged from 0.6 to 0.8 , most of the time during workdays. The comparison indicates the number of vehicles stored in link 1 during the school ending period kept traffic healthy after the adjustment. Thus, the issue of spillback was solved. 


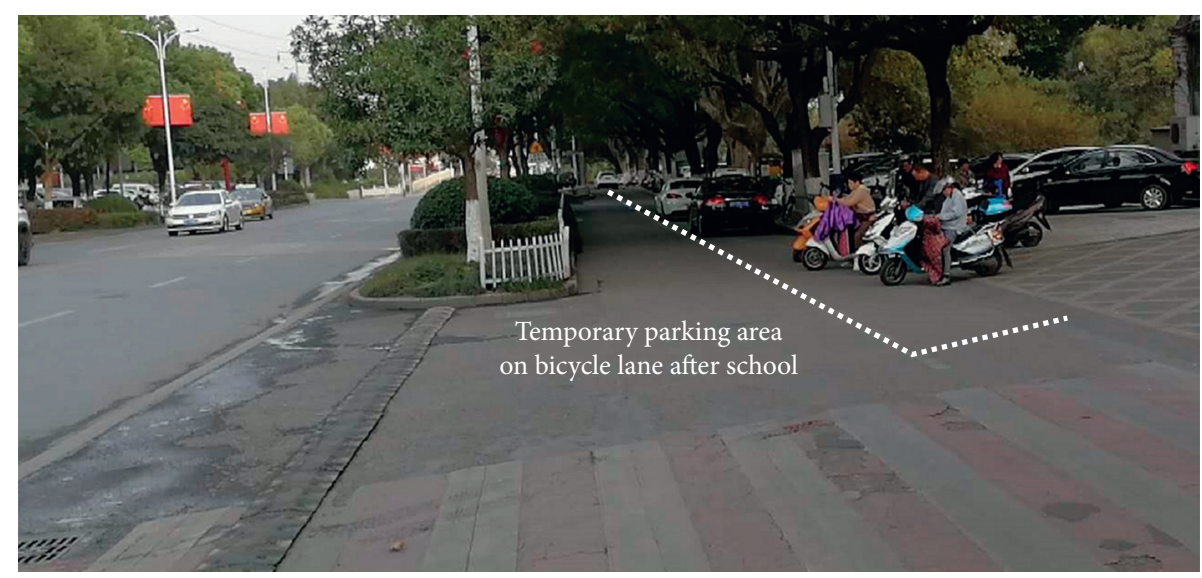

FIgURE 12: Adjustment in link 3 around the case school.

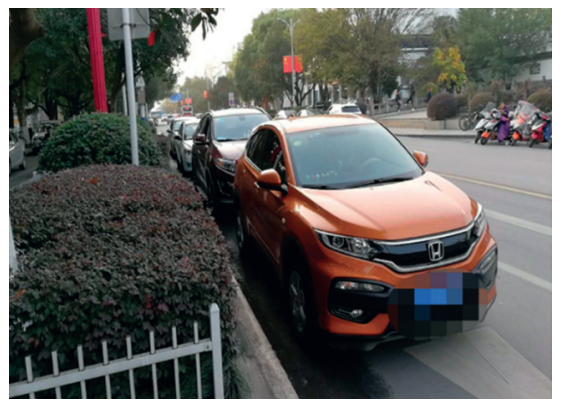

(a)

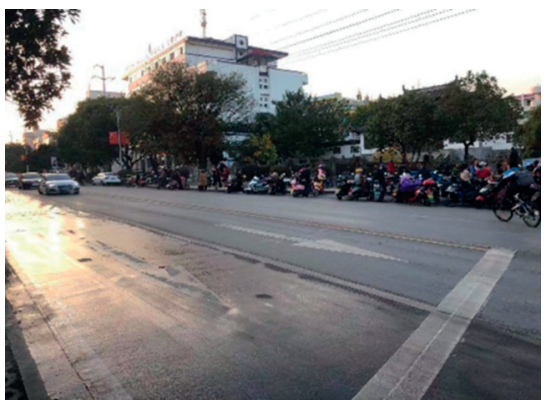

(b)

Figure 13: Field scene in front of the case school before and after fine-tuning. (a) 2019.10.17 16:30. (b) 2019.10.24 16:30.

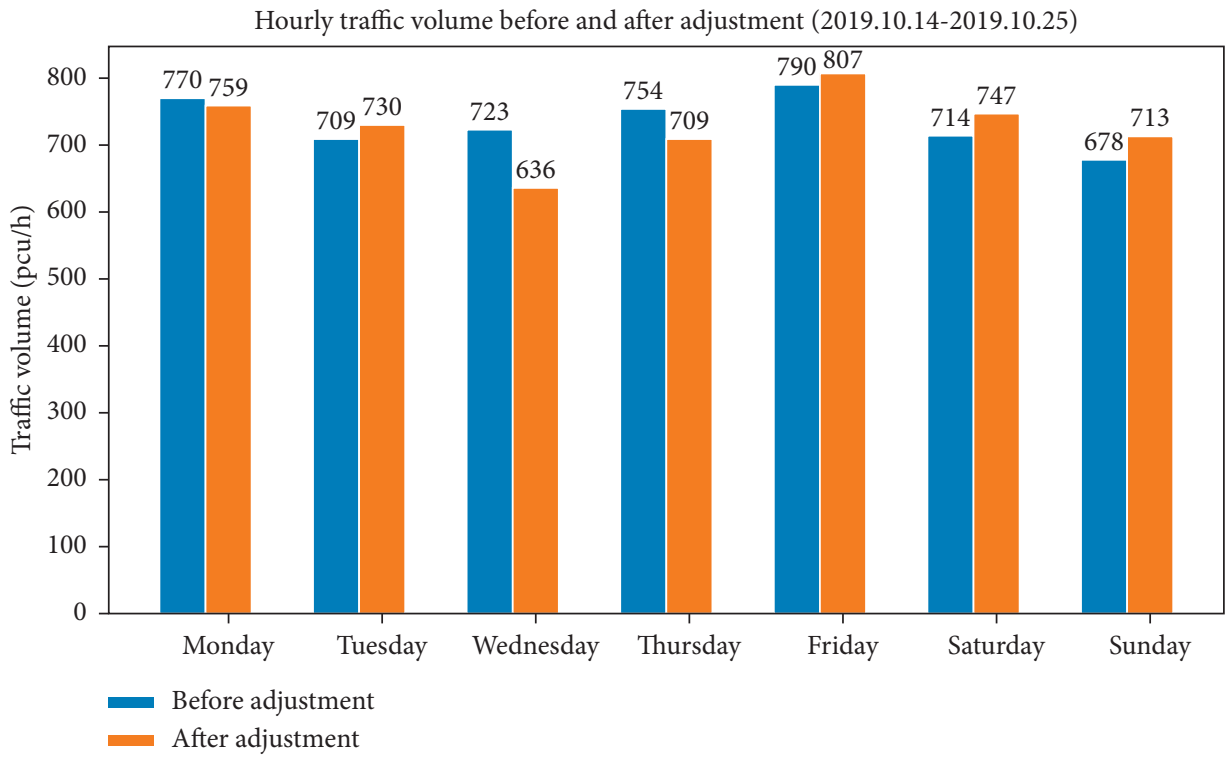

Figure 14: Traffic volume before and after adjustment in link 4. 

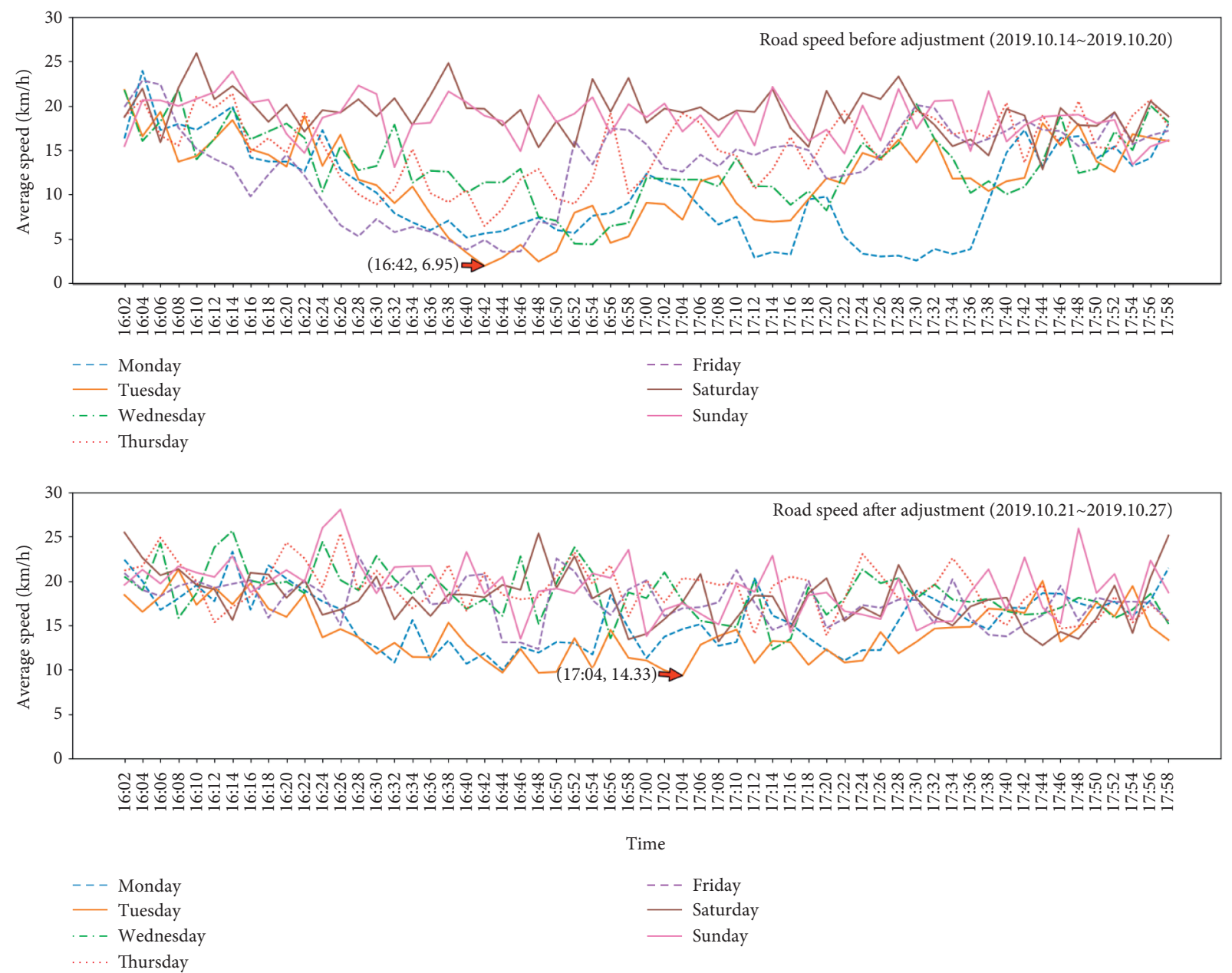

Figure 15: Road speed before and after adjustment in link 4 .

Moreover, all of the AVI data for high-frequency vehicles over the entire road network was used to reconstruct their trajectories during the school ending period and to evaluate individual vehicle's compliance situation of the mitigation strategies. We focused primarily on the following aspects of these high-frequency vehicles: (1) number of vehicles that were influenced by the adjustment implementation, (2) number of Type A vehicles that make detours as recommended, (3) number of Type $B$ vehicles that transform into Type A vehicles, and (4) number of Type B vehicles that disappear (drivers are no longer picking up their children). Table 3 presents the results of the trajectory analysis for high-frequency vehicles.

As shown in Table 4, positive effects were obtained from personalized management with different activity levels and resolutions for specific travellers. Overall, the number of high-frequency vehicles dropped by $37 \%$ from October 8 to November 30 (from 262 to 165). More than $65 \%$ of high-frequency vehicles' traffic behaviours were affected by proposed mitigation strategies. Most of Type A vehicles choose to detour according to the customized suggestion during the school ending period (detour number from 151 to 21 ). With the increase of the adjustment implementation time, the proportion of Type B vehicles that transformed into Type A vehicles increased significantly (from $19.6 \%$ to $60.7 \%$ ). The drivers of these vehicles are the beneficiaries of the adjustments. In addition to enjoying the convenience brought about by the improvement in traffic conditions, they are still able to pick up their children from school.

In brief, the proposed methodologies and personalized managements achieved good support with satisfactory stability for the PIAC alleviation around the case school. These were evident for complex scenarios with large traffic volume, limited land resources, and management budget. The adaptability of key vehicle clustering for specific travellers based on AVI data is considerably better than other congestion alleviation methods that mainly focused on the regional macrolevel. Therefore, the methodologies and experience presented in this study can provide a useful tool for relieving the PIAC around service centers in other regions faced with a similar situation. 

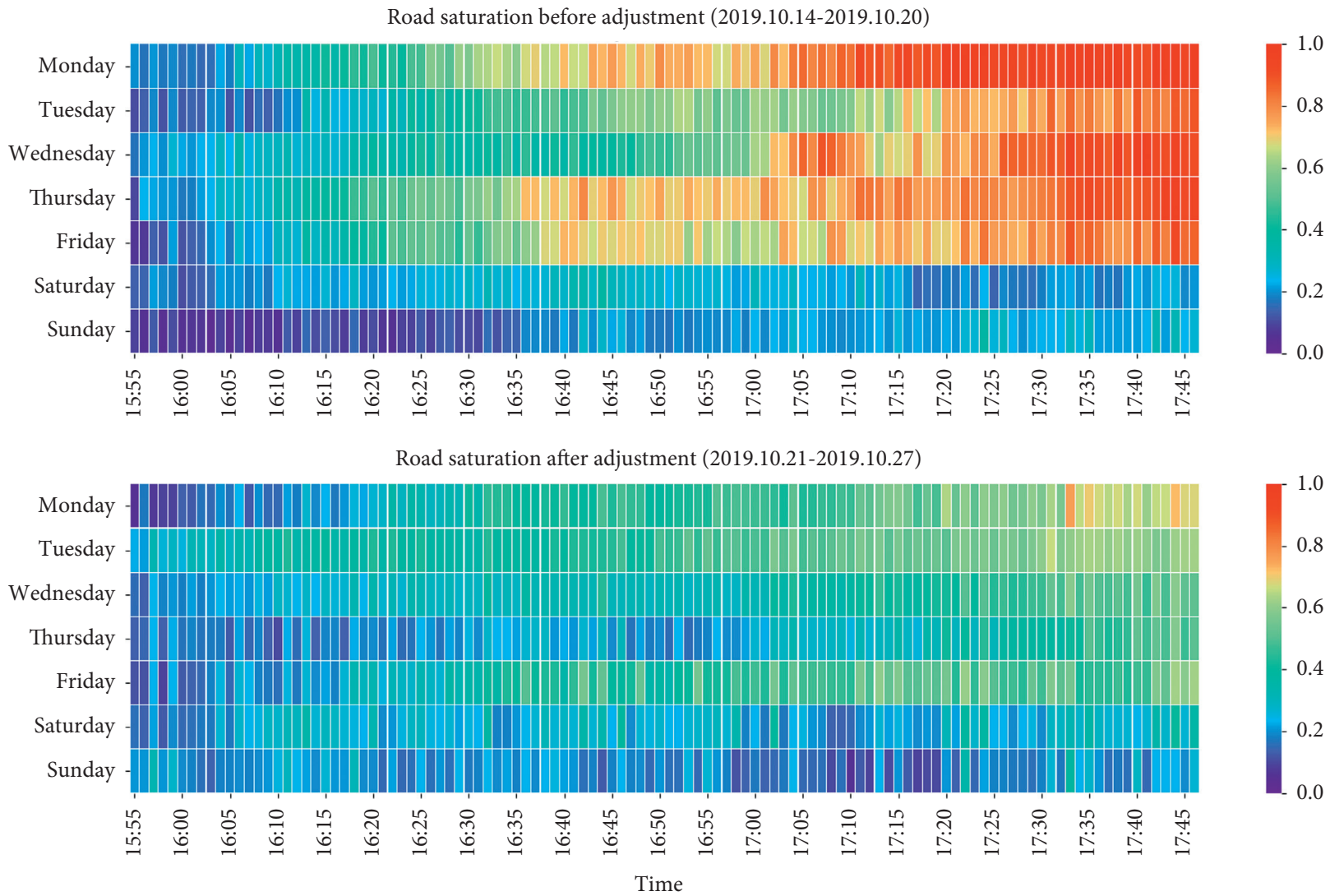

FIgURE 16: Road saturation before and after adjustment in link 1.

TABLE 4: High-frequency vehicles' compliance situation.

\begin{tabular}{lccccc}
\hline Month & Total number & Influence & Detour & Transform & Disappear \\
\hline October & 262 & 204 & 151 & 40 & 13 \\
November & 165 & 107 & 21 & 65 & 21 \\
\hline
\end{tabular}

\section{Conclusions}

In this paper, a data structure termed individual traffic behaviour image was designed to represent the long shortterm traffic profiles of individual travellers in the AVI data environment. A multilayer convolutional neural network was proposed and used to extract the long short-term traffic behaviour features. Then, a vehicle grading clustering framework based on behavioural characteristics was proposed for targeting the vehicles that were mainly responsible for the periodic impulsive aggregation congestion around the service centers. The objective of road management is updated under the influence of "relative perfect detection." Based on the AVI data for the road around the Xuancheng City 6th primary school, the periodic impulsive aggregation congestion was detected by reconstruction of parents' helpless curb parking behaviour during the school ending period. A framework for vehicle grading management was proposed and it became an important transport demand targeting model for formulating improvement schemes and mitigation strategies. The case study presented an effective approach to alleviating the periodic impulsive aggregation congestion around service centers through high-precision detection technologies. The comparison of traffic before and after adjustment was conducted and showed that school-related congestion can truly be relieved while avoiding additional construction. The high penetration rate of identity detection provides a new impetus for traffic planning and management in intelligent transportation systems. It allows us to clarify different transport demands for vehicles on the same section of the road and during the same period by considering their traffic behaviour characteristics. The regularly periodic impulsive aggregation of specific travellers for certain events is the root cause of the frequent local congestion around service centers. Formulating personalized managements with different activity levels and resolutions for specific travellers is a very important means of universal significance to solve the periodic impulsive aggregation congestion. Hence, the holistic and cooperative approach to the problem described here is cost-effective and maybe more generally applicable. 


\section{Data Availability}

The AVI data used to support the findings of this study were provided by the Xuancheng Traffic Police Division and cannot be made freely available. The data provided are for use only in this paper because it involves commercial and government requirements. Requests for access to these data should be made to Professor Zhi Yu, stsyuz@ mail.sysu.edu.cn.

\section{Conflicts of Interest}

The authors declare that there are no conflicts of interest regarding the publication of this study.

\section{Acknowledgments}

The authors would like to acknowledge the support funded by the National Natural Science Foundation of China (U1911401 and U1611461) and the National Key Research Program (2018YFB1601102).

\section{References}

[1] F. Liao, Q. Tian, T. Arentze, H.-J. Huang, and H. J. P. Timmermans, "Travel preferences of multimodal transport systems in emerging markets: the case of Beijing," Transportation Research Part A: Policy and Practice, vol. 138, pp. 250-266, 2020.

[2] G. Albert and D. Mahalel, "Congestion tolls and parking fees: a comparison of the potential effect on travel behavior," Transport Policy, vol. 13, no. 6, pp. 496-502, 2006.

[3] B. Dadashova, "Multivariate time series analysis of traffic congestion measures in urban areas as they relate to socioeconomic indicators," Socio-Economic Planning Sciences, vol. 75, Article ID 100877, 2021.

[4] Fu-Yu Wang, C.-M. Ye, and Y. Li, "Study on reasons and countermeasures of traffic congestion of urban road based on the queuing models," in Proceedings of the 2013 International Conference on Computational and Information Sciences, 2013.

[5] T. Nagatani, G. Ichinose, and K.-i. Tainaka, "Traffic jams induce dynamical phase transition in spatial rock-paperscissors game," Physica A: Statistical Mechanics and Its Applications, vol. 492, pp. 1081-1087, 2018.

[6] R. Xu, Influence Mechanism of Information Release Strategy on Urban Congestion Control Based on Traveler Behavior, Huazhong University of Science and Technology, Wuhan, China, 2019.

[7] X. Yu, S. Xiong, Y. He, W. E. Wong, and Y. Zhao, "Research on campus traffic congestion detection using BP neural network and Markov model," Journal of information security and applications, vol. 31, pp. 54-60, 2016.

[8] D. Matranga and F. Sapienza, "Congestion analysis to evaluate the efficiency and appropriateness of hospitals in Sicily," Health Policy, vol. 119, no. 3, pp. 324-332, 2015.

[9] J. Song, C. Zhao, S. Zhong, T. A. S. Nielsen, and A. V. Prishchepov, "Mapping spatio-temporal patterns and detecting the factors of traffic congestion with multi-source data fusion and mining techniques," Computers, Environment and Urban Systems, vol. 77, Article ID 101364, 2019.

[10] R. Cervero, "Road expansion, urban growth, and induced travel:A path analysis," Journal of the American Planning Association, vol. 69, no. 2, pp. 145-163, 2003.
[11] J.-F. Tsai and C.-P. Chu, "Analysis of the optimal length of road expansion-a case study of the Taipei metropolitan area," Transportation Research Part A: Policy and Practice, vol. 44, no. 3, pp. 147-158, 2010.

[12] L. J. Basso, F. Feres, and H. E. Silva, "The efficiency of bus rapid transit (BRT) systems: a dynamic congestion approach," Transportation Research Part B: Methodological, vol. 127, pp. 47-71, 2019.

[13] L. A. Guzman, J. Arellana, and V. Alvarez, "Confronting congestion in urban areas: developing Sustainable Mobility Plans for public and private organizations in Bogotá," Transportation Research Part A: Policy and Practice, vol. 134, pp. 321-335, 2020.

[14] X. Li and J.-Q. Sun, "Intersection multi-objective optimization on signal setting and lane assignment," Physica A: Statistical Mechanics and Its Applications, vol. 525, pp. 1233-1246, 2019.

[15] Y. Qi, D. Liu, X. Li, J. Lei, X. Xu, and Q. Miao, “An adaptive penalty-based boundary intersection method for many-objective optimization problem," Information Sciences, vol. 509, pp. 356-375, 2020.

[16] A. Aboudina and B. Abdulhai, "A bi-level distributed approach for optimizing time-dependent congestion pricing in large networks: a simulation-based case study in the Greater Toronto Area," Transportation Research Part C: Emerging Technologies, vol. 85, pp. 684-710, 2017.

[17] R. Li and M. Guo, "Effects of odd-even traffic restriction on travel speed and traffic volume: evidence from Beijing Olympic Games," Journal of Traffic and Transportation Engineering, vol. 3, no. 1, pp. 71-81, 2016.

[18] Z. He, G. Qi, L. Lu, and Y. Chen, "Network-wide identification of turn-level intersection congestion using only low-frequency probe vehicle data," Transportation Research Part C: Emerging Technologies, vol. 108, pp. 320-339, 2019.

[19] Z. Kan, L. Tang, M.-P. Kwan, C. Ren, D. Liu, and Q. Li, "Traffic congestion analysis at the turn level using Taxis' GPS trajectory data," Computers, Environment and Urban Systems, vol. 74, pp. 229-243, 2019.

[20] M. Kohan and J. M. Ale, "Discovering traffic congestion through traffic flow patterns generated by moving object trajectories," Computers, Environment and Urban Systems, vol. 80, Article ID 101426, 2020.

[21] C. Gutierrez, R. A. Lindor, O. Baker, D. Cutler, and J. D. Schuur, "State regulation of freestanding emergency departments varies widely, affecting location, growth, and services provided," Health Affairs, vol. 35, no. 10, pp. 1857-1866, 2016.

[22] Y. Xu and V. Ho, "Freestanding emergency departments in Texas do not alleviate congestion in hospital-based emergency departments," The American Journal of Emergency Medicine, vol. 38, no. 3, pp. 471-476, 2020.

[23] S. Zhang, "Relieve traffic congestion around the campus: the construction of the "under-ground pick-up system" in this elementary school in ningbo is remarkable," Auto \& Safety, vol. 8, pp. 78-81, 2019.

[24] E. Angelelli, I. Arsik, V. Morandi, M. Savelsbergh, and M. G. Speranza, "Proactive route guidance to avoid congestion," Transportation Research Part B: Methodological, vol. 94, pp. 1-21, 2016.

[25] R. Hu, L. Chen, and L. Zheng, "Congestion pricing and environmental cost at guangzhou baiyun international airport," Journal of Air Transport Management, vol. 70, pp. 126-132, 2018. 
[26] A. Zedgenizov and D. Burkov, "Methods for the traffic demand assessment based on the quantitative characteristics of urban areas functioning," Transportation Research Procedia, vol. 20, pp. 724-730, 2017.

[27] M. Lu, C. Sun, and S. Zheng, "Congestion and pollution consequences of driving-to-school trips: a case study in Beijing," Transportation Research Part D: Transport and Environment, vol. 50, pp. 280-291, 2017.

[28] A. Ermagun and D. Levinson, "Intra-household bargaining for school trip accompaniment of children: a group decision approach," Transportation Research Part A: Policy and Practice, vol. 94, pp. 222-234, 2016.

[29] T. D. Rhoulac, School Transportation Mode Choice and Potential Impacts of Automated Vehicle Location Technology, North Carolina State University, Raleigh, NC, USA, 2003.

[30] Z. Youting, Y. Zhi, and L. Xiying, "Evaluation methodology for license plate recognition systems and experimental results," IET Intelligent Transport Systems, vol. 12, no. 5, pp. 375-385, 2018.

[31] C. An, X. Guo, R. Hong, Z. Lu, and J. Xia, "Lane-based traffic arrival pattern estimation using license plate recognition data," IEEE Intelligent Transportation Systems Magazine, 2021.

[32] M. L. Tam Lam and W. H. K. Lam, "Application of automatic vehicle identification technology for real-time journey time estimation," Information Fusion, vol. 12, no. 1, pp. 11-19, 2011.

[33] Y. Zhu, Z. He, and W. Sun, "Network-wide link travel time inference using trip-based data from automatic vehicle identification detectors," IEEE Transactions on Intelligent Transportation Systems, vol. 21, no. 6, pp. 2485-2495, 2019.

[34] M. Cai, Z. Lan, Z. Zhang, and H. Wang, "Evaluation of road traffic noise exposure based on high-resolution population distribution and grid-level noise data," Building and Environment, vol. 147, pp. 211-220, 2019. 